\title{
Factores asociados con el uso de litotripsia mecánica en pacientes con cálculos gigantes de la vía biliar: ¿puede la dilatación papilar con balón grande disminuir su requerimiento?
}

\section{Can Endoscopic Papillary Large Balloon Dilation (EPLBD) Decrease the Need for Mechanical Lithotripsy in Patients with Giant Biliary Stones?}

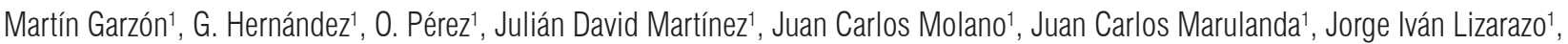 \\ Natan Hormaza', Mario Humberto Rey'.
}

Unidad de Gastroenterología y Endoscopia Digestiva, Hospital Universitario de la Samaritana (HUS) Universidad del Rosario, Bogotá D. C. (Colombia)

Fecha recibido: $25-10-16$ Fecha aceptado: $28-07-17$

\section{Resumen}

Introducción: cerca del $10 \%$ al $15 \%$ de los pacientes con coledocolitiasis presentan cálculos gigantes, definidos como aquellos que miden más de $10 \mathrm{~mm}$ a $15 \mathrm{~mm}$. La extracción de estos puede ser problemática, aun para endoscopistas experimentados. Casi todos los pacientes con esta patología pueden ser tratados endoscópicamente, y la mayoría de los cálculos son removidos con la realización de esfinterotomía (EFT), litotripsia mecánica (LM) o dilatación papilar con balón grande (DPBG). La DPBG ha demostrado ser efectiva en el manejo del cálculo gigante y disminuye la necesidad de LM.

Objetivos: determinar la prevalencia de coledocolitiasis gigante en el Hospital Universitario de la Samaritana (HUS) entre el 2009 y el 2014, la frecuencia de éxito endoscópico y la necesidad de manejo quirúrgico, además de evaluar los factores asociados con el uso de litotripsia en casos de cálculos gigantes de la vía biliar.

Materiales y métodos: el presente es un estudio de casos y controles de pacientes con cálculos gigantes, definidos como cálculos mayores de $10 \mathrm{~mm}$ en pacientes llevados a colangiopancreatografía retrógrada endoscópica (CPRE) en el HUS entre 2009 y 2014. Los casos fueron los pacientes a quienes se les realizó litotripsia y, los controles, quienes no requirieron este procedimiento. Los resultados se presentan como medias o medianas, y se indican sus respectivas medidas de dispersión para las variables continuas, según pruebas de normalidad o como frecuencias y porcentajes para las variables categóricas. Se realizó un análisis de regresión logística con el principal objetivo de determinar los factores asociados con litotripsia, en el que se incluyeron las variables biológicamente plausibles o que, estadísticamente, mostraron diferencias en el análisis bivariado $(p<0,200)$. Los resultados se presentan como odds ratios (oportunidad relativa) ajustados (ORA).

Resultados: entre 2009 y 2014, se realizaron un total de 1403 CPRE, de las cuales $198(14,1 \%)$ tuvieron presencia de cálculos gigantes. Fue más frecuente la coledocolitiasis gigante en el sexo femenino que en el masculino. La edad promedio de los pacientes fue de 66,6 años. El diámetro promedio del colédoco fue de 18 $\mathrm{mm}$, y el diámetro promedio de los cálculos fue de $18 \mathrm{~mm}$. Al $99 \%$ de los pacientes se les realizo EFT, el $28 \%$ requirió $L M$, al $48 \%$ se les realizo DPBG, el 5,5\% requirió LM y DPBG, y el 30,8\% requirió el avance de prótesis biliar y más de una CPRE para la resolución de la coledocolitiasis. En el $89,9 \%$ de los casos, el manejo endoscópico fue exitoso. El 10,1\% requirió cirugía. En el $9,0 \%$ de los casos, se presentaron complicaciones ( 6 pancreatitis, 7 sangrados, 2 impactaciones de canastilla y 1 perforación). En el análisis bivariado, la DPBG fue un factor protector del requerimiento de LM (ORA 0,07-IC del 95\% 0,025-0,194), y el tamaño del colédoco es un predictor del requerimiento de $L M(p<0,05)$.

Conclusiones: en nuestra serie, la prevalencia de coledocolitiasis gigante y el éxito del manejo endoscópico es similar a la reportada en la literatura. La EFT, la LM y la DPBG fueron efectivas en el manejo de los cálculos gigantes. El tamaño del colédoco es un predictor de requerimiento de LM y la DPBG disminuyó el requerimiento de LM.

\section{Palabras claves}

Coledocolitiasis gigante, litotripsia mecánica, dilatación papilar balón grande. 


\begin{abstract}
Introduction: About $10 \%$ to $15 \%$ of patients with choledocholithiasis develop a giant calculus, defined as one that measures more than $10 \mathrm{~mm}$ to $15 \mathrm{~mm}$. Removal of these stones can be problematic even for experienced endoscopists. Almost all patients with this pathology can be treated endoscopically: most are removed with sphincterotomies, mechanical lithotripsy (ML) or large balloon papillary dilatation (LBPD). LBPD has been shown to be effective in handling giant calculi and decreases the need for ML.

Objectives: The aim of this study was to determine the prevalence of giant choledocholithiasis at HUS between 2009 and 2014, the frequency of successful endoscopic treatment, and the frequency of surgery in these cases. We also evaluated factors associated with the use of lithotripsy to remove giant biliary calculi.

Materials and Methods: This study is a case-control study of patients who had giant calculi and underwent ERCP at the HUS between 2009 and 2014. Calculi larger than $10 \mathrm{~mm}$ were defined as giant. Cases were patients who underwent lithotripsy while controls were those who did not require lithotripsy. The results for continuous variables are presented as means or medians and their respective measures of dispersion while results for categorical variables are presented according to tests of normality or as frequencies and percentages. A logistic regression analysis was used to determine factors associated with lithotripsy. Biologically plausible variables and those that had statistically significant differences in the bivariate analysis $(p<0.200)$ were included. The results are presented as adjusted odds ratios (ORA).

Results: Between 2009 and 2014, a total of 1403 ERCPs were performed. Giant calculi were found in 198 $(14.1 \%)$ of these procedures. Giant choledocholithiasis was more common in female patients than in male patients. The patients' mean age was 66.6 years. The average diameter of the common bile duct was 18 $\mathrm{mm}$, and the average diameter of the stones was $18 \mathrm{~mm}$. Ninety-nine percent of these patients underwent sphincterotomies, $28 \%$ required $\mathrm{ML}$, $48 \%$ underwent LBPD, 5.5\% required $\mathrm{ML}$ and $\mathrm{LBPD}$, and $30.8 \%$ required biliary stents and more than one ERCP for the resolution of choledocholithiasis. In $89.9 \%$ of cases, endoscopic management was successful. Only $10.1 \%$ of the total number of patients required surgery. Complications occurred in $9.0 \%$ of the cases: 6 cases of pancreatitis, 7 cases of bleeding, 2 impacted cannula and 1 perforation. In the bivariate analysis, LBPD was a protective factor against the need for ML (ORA 0.07-IC 95\% $0.025-0.194)$ and choledochal size was a predictor a need for MM $(p<0.05)$.

Conclusions: In our series the prevalence of giant choledocholithiasis and the success of endoscopic management are similar to those reported in the literature. Sphincterotomies, ML and LBPD were effective for managing giant calculi. Choledochal size is a predictor of a need for ML while performance of LBPD decreased the need for ML.

\section{Keywords}

Choledocholithiasis, giant calculi, mechanical lithotripsy, large balloon papillary dilation.
\end{abstract}

\section{INTRODUCCIÓN}

La enfermedad litiásica biliar afecta a más de 20 millones de adultos en Norteamérica, con un costo anual de 6,2 miles de millones de dólares (1). Aproximadamente el $85 \%$ al $90 \%$ de los cálculos biliares pueden extraerse con un balón o una canastilla después de realizar una esfinterotomía o una dilatación papilar con balón (DPB) $(1,2)$. Cerca del $10 \%$ al $15 \%$ de los pacientes con coledocolitiasis presentan un cálculo gigante definido como aquel que mide más de $10 \mathrm{~mm}$ a $15 \mathrm{~mm}$ (Figura 1). La extracción de estos puede ser problemática aun para endoscopistas experimentados. Casi todos los pacientes con esta patología pueden ser tratados endoscópicamente, y la mayoría de los cálculos se extraen con la realización de una esfinterotomía (EFT), litotripsia mecánica (LM) o dilatación papilar con balón grande (DPBG) $(1,3,4,5)$.

La LM fue descrita en 1982 y ha sido históricamente la más utilizada para el manejo y la extracción de los cálcu- los gigantes, con unas tasas de éxito del 79\% al 92\%. Las fallas en la extracción de cálculos con esta técnica se deben a impactación del cálculo en el conducto biliar común, y los cálculos mayores de $2 \mathrm{~cm}$ son predictores de falla. La incidencia de complicaciones con su uso oscila entre el 6\% y el $13 \%$, y la pancreatitis y el sangrado son las más frecuentes $(1,5)$. Nuestra tasa de éxito reportada previamente con LM fue del $89 \%$, y el tamaño del cálculo $>24 \mathrm{~mm}$ fue igualmente predictor de fracaso (6).

La DPBG se introdujo como una alternativa a la EFT para pacientes con cálculos pequeños a moderados $(4 \mathrm{~mm}$ a $10 \mathrm{~mm}$ ). Sin embargo, este procedimiento está asociado con un alto riesgo de pancreatitis y necesidad adicional de LM $(7,8)$. La DPBG después de una EFT limitada o pequeña fue descrita en 2003 por Ersoz y colaboradores para la remoción de cálculos $>10 \mathrm{~mm}$ o múltiples cálculos biliares. Ha demostrado ser efectiva en el manejo de cálculos gigantes y, según algunos estudios, disminuye la necesidad de $\operatorname{LM}(3,4,8)$. Este método combina las ventajas de 


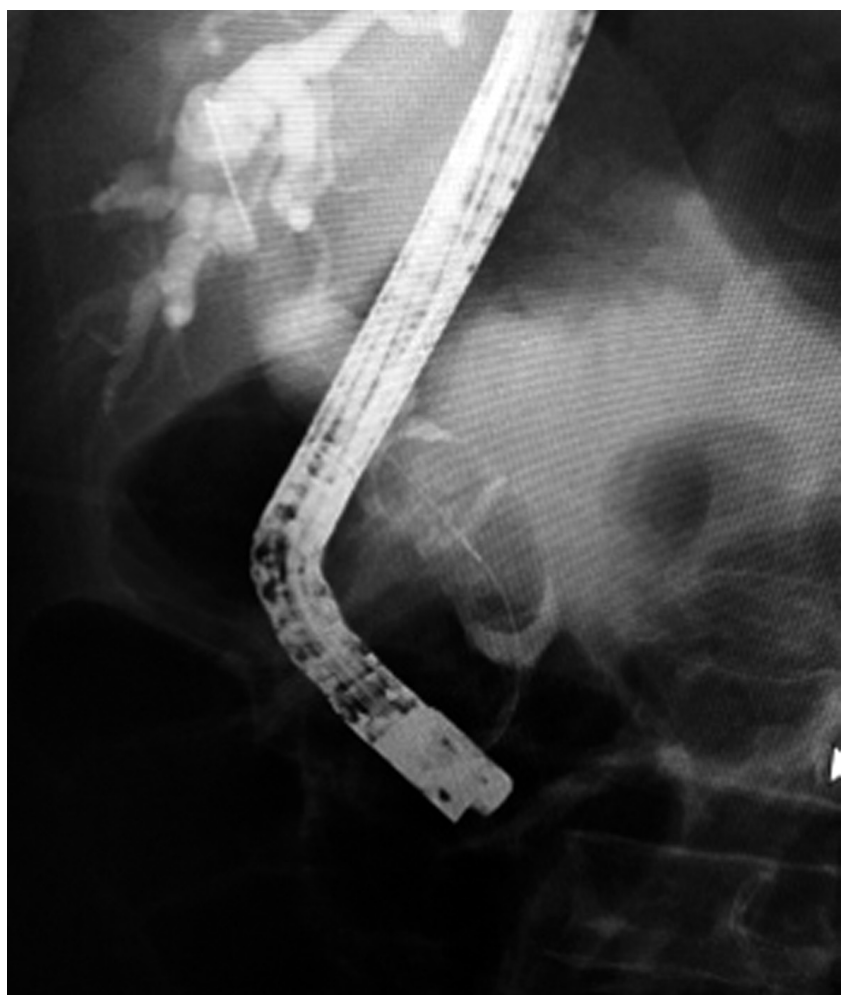

Figura 1. Cálculo biliar gigante.

la EFT y la DPB y aumenta la tasa de extracción de cálculos mientras disminuye las complicaciones de la EFT o la DPB solas (7). Se realiza utilizando balones de $12 \mathrm{~mm}$ a $20 \mathrm{~mm}$ y lleva a una apertura papilar mayor a la obtenida con la EFT o la DPB solas, lo que permite la extracción de los cálculos sin la necesidad de LM y, por esto, acorta el tiempo del procedimiento y de irradiación $(1,8,9,10,11,12)$.

\section{OBJETIVOS}

El objetivo del presente estudio fue determinar la prevalencia de la coledocolitiasis con cálculos gigantes en el HUS entre 2009 y 2014, la frecuencia de éxito del manejo endoscópico, la necesidad de manejo quirúrgico y evaluar los factores asociados con el uso de la litotripsia en cálculos gigantes de la vía biliar.

\section{MATERIALES Y METODOS}

Se revisaron en forma retrospectiva los registros clínicos de los pacientes llevados a colangiopancreatografía retrógrada endoscópica (CPRE) durante el período comprendido entre enero de 2009 y diciembre de 2014 . Se calculó la prevalencia de la coledocolitiasis con cálculos gigantes, defini- dos como cálculos mayores de $10 \mathrm{~mm}$. Se realizó un estudio retrospectivo de casos y controles. Los casos fueron los pacientes a quienes se les realizó litotripsia y, los controles, quienes no requirieron este procedimiento. Los procedimientos los realizaron los gastroenterólogos del servicio del Hospital Universitario de la Samaritana, centro de referencia departamental de cuarto nivel del departamento de Cundinamarca (Colombia), con 2 duodenoscopios marca Olympus (GIF-Q180V y TJF-Q180V). Los resultados se presentan como medias o medianas, y se indican sus respectivas medidas de dispersión para las variables continuas, según pruebas de normalidad, o como frecuencias y porcentajes para las variables categóricas. Se realizó un análisis de regresión logística con el principal objetivo de determinar los factores asociados con litotripsia, en el que se incluyeron las variables biológicamente plausibles o que, estadísticamente, mostraron diferencias en el análisis bivariado $(\mathrm{p}<0,200)$. Los resultados se presentan como odds ratios (oportunidades relativas) ajustados (ORA).

\section{RESULTADOS}

Entre 2009 y 2014, se realizaron un total de 1403 CPRE, de las cuales $198(14,1 \%)$ tuvieron presencia de cálculos gigantes. La edad promedio fue de 66,67 años. El 70,7\% fueron mujeres y el $29,3 \%$ fueron hombres. La mediana del diámetro del colédoco fue de $18 \mathrm{~mm}$, con un rango intercuartílico (RIQ) de entre 15 y $20 \mathrm{~mm}$. La Tabla 1 resume los hallazgos de la población total. Además del grupo de pacientes a los que se les realizó litotripsia, se muestra la tasa de fallo y litotripsia parcial.

Tabla 1. Características generales de los pacientes incluidos.

\begin{tabular}{lc}
\hline \multicolumn{1}{c}{ Característica } & Valor \\
\hline Edad, años (media $\pm \mathrm{DE})$ & $66,68 \pm 15,71$ \\
Tamaño del colédoco, mm (mediana, RIQ) & $18(16-20)$ \\
Diámetro mayor del cálculo, mm (mediana, RIQ) & $18(15-21)$ \\
Diámetro menor del cálculo, mm (mediana, RIQ) & $15(13-18)$ \\
Bilioplastía (DPBG), n/N (\%) & $95 / 198(48,0)$ \\
Papilotomía, n/N (\%) & $196 / 198(99,0)$ \\
Balón, n/N (\%) & $122 / 198(61,6)$ \\
Canastilla, n/N (\%) & $164 / 198(82,8)$ \\
Prótesis (stent), n/N (\%) & $61 / 198(30,8)$ \\
Cirugía, n/N (\%) & $19 / 198(10,1)$ \\
Litotripsia, n/N (\%) & $56 / 198(28,3)$ \\
Litotripsia fallida, n/N (\%) & $7 / 56(12,5)$ \\
Litotripsia parcial, n/N (\%) & $5 / 56(8,9)$ \\
\hline
\end{tabular}

DE: desviación estándar; RIQ: rango intercuartílico. 
El diámetro promedio del colédoco fue de $18 \mathrm{~mm}$ y el diámetro promedio de los cálculos también fue de $18 \mathrm{~mm}$. $\mathrm{Al}$ 99\% de los pacientes se les realizó EFT, el 28\% requirió $\mathrm{LM}$, al 48\% se les realizó $\mathrm{DPBG}$, el 5,5\% requirió LM y DPBG, y el 30,8\% requirió el avance de prótesis biliar y más de una CPRE para la resolución de la coledocolitiasis. En el $89,9 \%$ de los casos, el manejo endoscópico fue exitoso. El $10,1 \%$ requirió cirugía. En el 9,0\% de los casos, se presentaron complicaciones que incluyeron: 6 pancreatitis $(3,0 \%$, todas leves), 7 sangrados del área de la papilotomía (3,5\%, ninguno requirió manejo quirúrgico), 2 impactaciones de canastilla ( $1,0 \%$, ambas en pacientes con LM, una resuelta con el litotriptor de urgencia y, la otra, con requerimiento de cirugía) y 1 perforación $(0,5 \%)$ que recibió manejo médico conservador. No se presentaron mortalidades asociadas con el procedimiento.

Para el análisis bivariado, se evaluó si la bilioplastía se comporta como factor protector del requerimiento de litotripsia. En el análisis bivariado la DPBG fue un factor protector del requerimiento de LM (ORA 0,07-IC del 95\% $0,025-0,194)$, y el tamaño del colédoco es un predictor del requerimiento de $\operatorname{LM}(\mathrm{p}<0,05)$.

Las Tablas 2 y 3 presentan los resultados del análisis bivariado, con sus respectivos OR crudos, y el resultado de la regresión logística. El análisis se ajustó según las demás variables incluidas en el estudio, susceptibles de confusión o interacción, y las que mostraron una diferencia significativa en el bivariado.

\section{DISCUSIÓN}

Cerca del 10\% al 15\% de los pacientes con coledocolitiasis presentan un cálculo gigante. La extracción de estos puede ser problemática aun para endoscopistas experimentados. En nuestra serie, la prevalencia de cálculos gigantes en los pacientes llevados a CPRE por coledocolitiasis fue del $14,1 \%(\mathrm{n}=198)$, cifra similar a la reportado en la literatura. Nuestra tasa de éxito con manejo endoscópico fue del
$89,9 \%$, y solo el $10,1 \%$ de los pacientes requirieron manejo quirúrgico. Se le realizó LM al 28,3\%, y la tasa de éxito con la LM fue del 89\% (reportada previamente). Desde el 2011, año en el que iniciamos nuestra experiencia con la DPBG, se realizó DPBG al 48\% de los pacientes; desde su inicio, disminuyó nuestra necesidad de LM, y solo el 5,5\% de los pacientes requirieron DPBG y LM. El tamaño del colédoco fue un factor predictor de la necesidad de LM.

La DPBG después de una EFT limitada o pequeña fue descrita en 2003 por Ersoz y colaboradores para la remoción de cálculos $>10 \mathrm{~mm}$ o múltiples cálculos biliares $(1,8$, $9,12)$. Se utiliza para crear una mayor apertura del orificio biliar con un balón de diámetro grande ( $12 \mathrm{~mm}$ a $20 \mathrm{~mm}$ ) para simplificar la remoción de cálculos gigantes y difíciles del conducto biliar y como una alternativa a la LM, ya que esta consume tiempo, puede generar impactación o fractura de la canastilla de Dormia y aumentar el riesgo de eventos adversos. La EFT es inicialmente recomendada como paso previo a la DPBG, ya que se cree que está asociada con una disminución del riesgo de pancreatitis posprocedimiento (Figura 2) $(8,9,13,14)$. Puede utilizarse como método inicial cuando se documentan cálculos gigantes en las imágenes o cuando la remoción convencional con EFT y canastilla han fallado $(14,15,16)$.

Los factores de riesgo asociados con el procedimiento que pueden estar relacionados con la presencia de eventos adversos incluyen el tamaño de la EFT, el diámetro del balón utilizado y la forma de inflar el balón $(8,9,14)$. El diámetro del conducto biliar distal es el factor más importante al seleccionar el diámetro del balón que se va a utilizar, ya que la dilatación más allá del diámetro del colédoco aumenta el riesgo de perforación y, por ende, el diámetro máximo de inflado del balón no debe exceder el diámetro mayor del colédoco distal. Aunque el diámetro de los balones para DPBG oscila de $12 \mathrm{~mm}$ a $20 \mathrm{~mm}$, en la mayoría de los casos se utilizan balones de $12 \mathrm{~mm}$ a $15 \mathrm{~mm}$ para prevenir eventos adversos (Figura 3). El inflado rápido y forzado del balón a través de un conducto biliar distal

Tabla 2. Análisis bivariado.

\begin{tabular}{|c|c|c|c|c|}
\hline \multirow[t]{2}{*}{ Variable } & \multicolumn{2}{|c|}{ Litotripsia } & \multirow[t]{2}{*}{$\mathrm{p}$} & \multirow[t]{2}{*}{ OR (IC del 95\%) } \\
\hline & Sí & No & & \\
\hline Edad, años (media $\pm \mathrm{DE}$ ) & $66,86 \pm 14,5$ & $66,61 \pm 16,20$ & $0,920^{*}$ & $\mathrm{NC}$ \\
\hline Tamaño del colédoco, mm (mediana, RIQ) & $19,0(17,0-22,0)$ & $18,0(15,0-20,0)$ & $0,013^{\wedge}$ & $\mathrm{NC}$ \\
\hline Diámetro mayor del cálculo, mm (mediana, RIQ) & $19,0(15,0-23,0)$ & $18,0(15-20)$ & $0,149^{\wedge}$ & $\mathrm{NC}$ \\
\hline Diámetro menor del cálculo, mm (mediana, RIQ) & $15,0(14,0-18,5)$ & $15,0(13,0-18,0)$ & $0,394^{\wedge}$ & $\mathrm{NC}$ \\
\hline Sexo masculino, $\mathrm{n} / \mathrm{N}(\%)$ & $16(27,6)$ & $42(72,4)$ & $0,889^{+}$ & $0,952(0,481-1,885)$ \\
\hline Bilioplastía, $\mathrm{n} / \mathrm{N}(\%)$ & $5(5,3)$ & $90(94,7)$ & $0,000^{+}$ & $0,057(0,021-0,151)$ \\
\hline
\end{tabular}

*T de Student para diferencia de medias; ${ }^{\wedge} \mathrm{U}$ de Mann-Whitney. ${ }^{+} X^{2}$ 
Tabla 3. Variables asociadas con el desenlace de requerimiento de litotripsia.

\begin{tabular}{lcc}
\hline \multicolumn{1}{c}{ Variable } & ORA (IC del 95\%) & p \\
\hline Edad & $0,998(0,972-1,023)$ & 0,857 \\
Sexo masculino & $0,619(0,268-1,422)$ & 0,619 \\
Tamaño del colédoco & $1,117(1,004-1,242)$ & 0,041 \\
Diámetro mayor del cálculo & $0,996(0,932-1,065)$ & 0,908 \\
Bilioplastía & $0,070(0,025-0,194)$ & 0,000 \\
\hline
\end{tabular}

ORA: odds ratio (oportunidad relativa) ajustado.

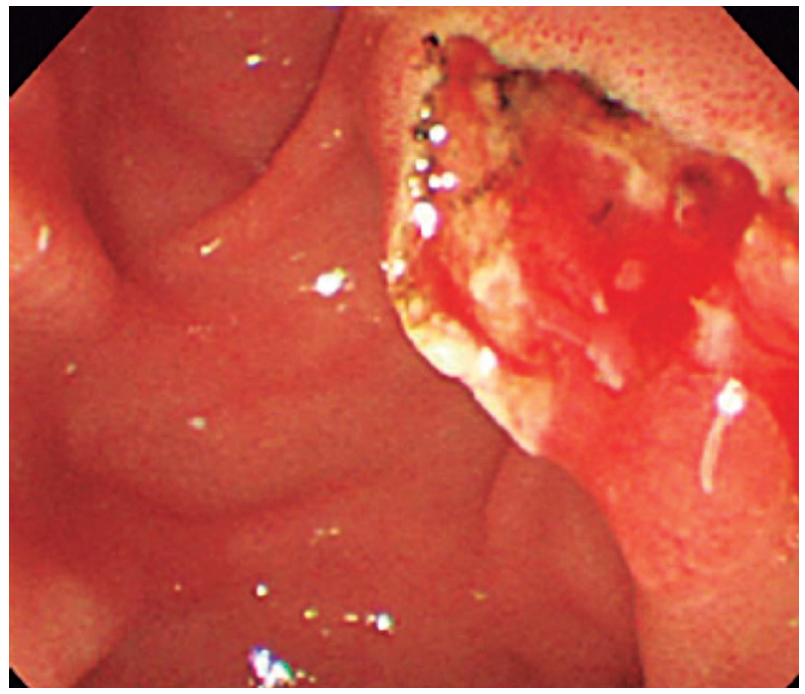

Figura 2. Esfinterotomía mediana.

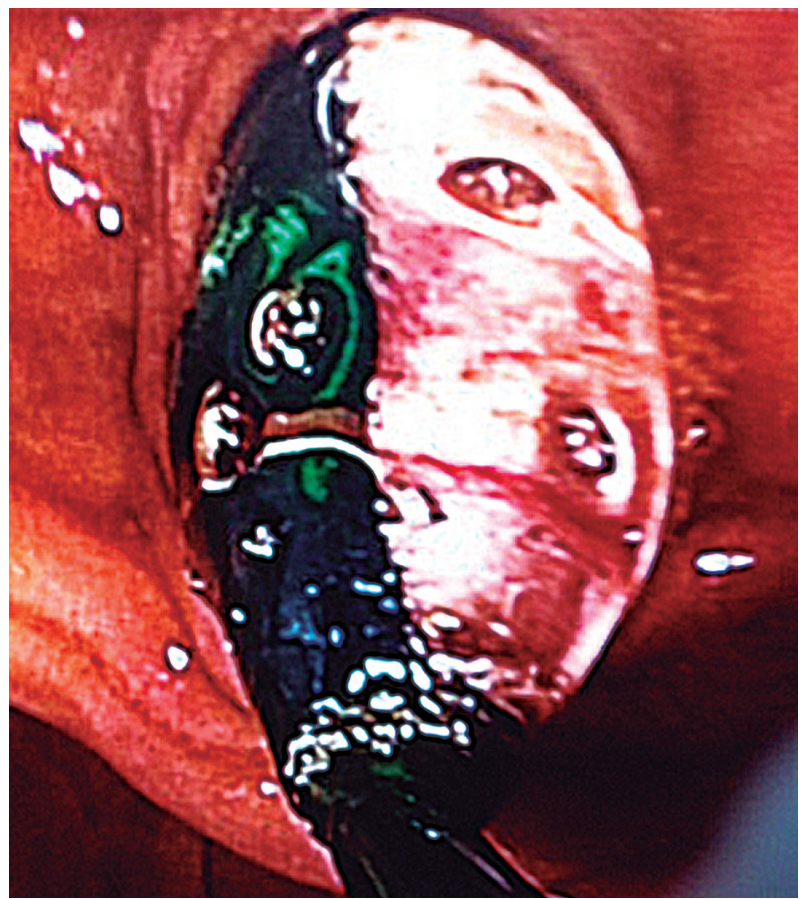

Figura 3. Dilatación papilar con balón grande (DPBG). estrecho puede llevar a perforación y sangrado. Por esto, el balón siempre debe inflarse lenta y gradualmente hasta que desaparezca su cintura (Figura 4). Si la cintura del balón no desaparece, puede sugerir la presencia de una estenosis biliar oscura (oculta), y no se debe dilatar por encima de este nivel. En ese caso, se debe desinflar el balón para evitar el riesgo de complicaciones. En los pacientes con estenosis biliares evidentes, o en conductos no dilatados, la DPBG no se recomienda debido al riesgo de perforación (17, 18). La duración usual de la DPBG es de 30 a 60 segundos después de desaparecer la cintura, aunque en diferentes estudios respecto del tiempo de dilatación oscilan entre los 10 segundos y los 180 segundos $(14,18)$; se requieren estudios adicionales para establecer la duración óptima de la dilatación. En nuestra serie, el tiempo de dilatación fue de 60 segundos.

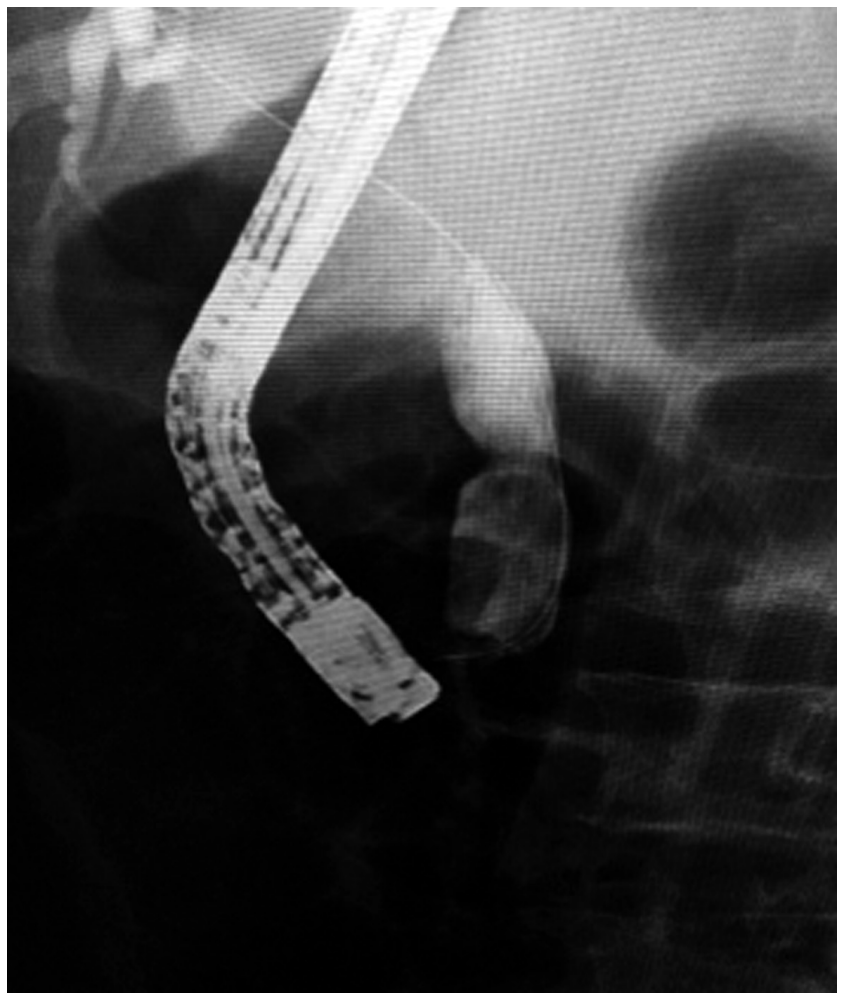

Figura 4. Dilatación (cintura del balón).

La LM ha demostrado ser una técnica que consume tiempo y está relacionada con eventos adversos como impactación de la canastilla y lesión del conducto biliar (14). Se ha propuesto que el orificio papilar amplio creado con la DPBG facilita la extracción de cálculos grandes y reduce la necesidad de LM. En diferentes metaanálisis, fue evidente que la DPBG redujo la necesidad de LM en comparación con la EFT sola $(8,19,20,21)$, aunque otros no han reportado ninguna diferencia significativa (22) y 
consideran que esta afirmación es solo una especulación. En nuestra serie, la DPBG fue un fuerte factor protector del uso de LM, tanto en el análisis multivariado como en la regresión logística.

En cuanto a las complicaciones, la tasa global de eventos adversos (pancreatitis, sangrado y perforación) es más baja para la DPBG que para la EFT sola, lo cual fue observado en 4 metaanálisis que evaluaron una comparación de DPBG más EFT con EFT sola (14, 19, 21, 22, 23). En un estudio multicéntrico de casos y controles a gran escala de 946 pacientes, el análisis de los subgrupos mostró que los cálculos mayores de $16 \mathrm{~mm}$, la presencia de cirrosis y la EFT completa (grande) fueron factores independientemente asociados con la presencia de eventos adversos. Adicionalmente, la DPBG no aumenta el riesgo de pancreatitis y, en una revisión sistemática de estudios de DPBG (14), la pancreatitis ocurrió en el 2,4\% de los pacientes que recibieron DPBG y EFT. Un posible mecanismo de reducción en la tasa de pancreatitis se debe a que la fuerza radial ejercida durante la dilatación con EFT se dirige hacia el conducto biliar, lejos del orificio pancreático, lo que produce una lesión periampular menor alrededor del conducto pancreático $(13,14)$. Sin embargo, se cree que la EFT tiene un papel limitado en prevenir la pancreatitis en los pacientes con DPBG, ya que los pacientes con DPBG sin EFT no tienen un aumento en el riesgo de pancreatitis. Por esto, se ha sugerido otra hipótesis acerca del mecanismo de la pancreatitis, la que postula que la frecuencia de manipulación con canastilla de Dormia y balón de extracción - tanto en DPBG con y sin EFT- es reducida debido a que el orificio papilar es lo suficientemente amplio (Figura 5), lo que resulta en menos trauma periampular, edema y más bajo riesgo de pancreatitis, y esto explica por qué el riesgo de lesión del orificio ampular con la dilatación papilar con balones de menor calibre $(<10 \mathrm{~mm})$ aumenta debido al paso de los instrumentos para extracción de los cálculos por un orificio inadecuadamente ampliado (14).

En nuestra serie, la tasa de complicaciones fue acorde a lo reportado en la literatura, con 6 pancreatitis $(3,0 \%$, todas leves), 2 impactaciones de canastilla $(1,0 \%)$-ambas en pacientes con $\mathrm{LM}-\mathrm{y}$ una perforación $(0,5 \%)$ no asociada con la DPBG, sino presentada con la aleta de un stent biliar (el paciente recibió manejo conservador).

\section{CONCLUSIONES}

La prevalencia de cálculos gigantes en nuestra institución y la tasa de éxito del manejo endoscópico son similares a las reportadas en la literatura. La DPBG con EFT mediana es un procedimiento seguro, y la DPBG es un fuerte factor protector del uso de litotripsia, lo que podría reducir el

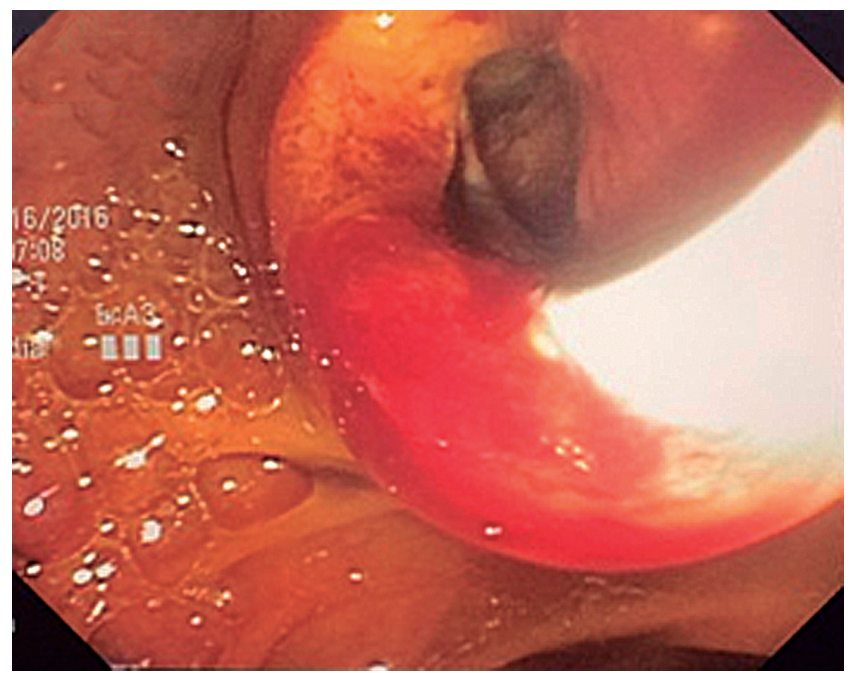

Figura 5. Orificio papilar posdilatación.

tiempo endoscópico, las complicaciones derivadas de esta terapia y los costos adicionales.

\section{REFERENCIAS}

1. ASGE. The role of endoscopy in the management of choledocholithiasis. Gastrointestinall Endoscopy. 2011;74(4):73144. Doi: https://doi.org/10.1016/j.gie.2011.04.012

2. Hwang JC, Kim JH, Lim SG, et al. Endoscopic large-balloon dilation alone vs endoscopic sphincterotomy plus largeballoon dilatation for the treatment of large bile duct stones. BMC Gastroenterology. 2013;13(15):1-6.

3. Artifon E. Cálculo difícil en CPRE. Rev Col Gastroenterol. 2011;26(2):80-1.

4. Vargas R, Guzmán G. Dilatación papilar con balón grande para coledocolitiasis. Rev Col Gastroenterol. 2011;26(2):94-9.

5. Stefanidis G, Christodoulou C, Manolakopoulos S, et. al. Endoscopic extraction of large common bile duct stones: a review article. World J Gastrointest Endosc. 2012;4(5):16779. Doi: https://doi.org/10.4253/wjge.v4.i5.167

6. Garzón MA, Pinto R, Martínez JD, et al. Coledocolitiasis gigante y factores predictores de éxito y fracaso del manejo endoscópico. POSTER ACADI (Barranquilla). 2013.

7. Fu BQ, Xu YP, Toa LS, et al. Endoscopic papillary balloon intermittent dilatation and endoscopic sphincterotomy for bile duct stones. World J Gastroenterol. 2013;19(15):242532. Doi: https://doi.org/10.3748/wjg.v19.i15.2425

8. Sakai Y, Tsuyuguchi T, Kawaguchi Y, et al. Endoscopic papillary large balloon dilation for removal of bile duct stones. World J Gastroenterol. 2014;20(45):17148-54. Doi: https://doi.org/10.3748/wjg.v20.i45.17148

9. Guo S, Meng H, Duan Z, et al. Small sphincterotomy combined with endoscopic papillary large balloon dilation vs 
sphincterotomy alone for removal of common bile duct stones. World J Gastroenterol. 2014;20(47):17962-9.

10. Dong SH. Highlights of pancreatobiliary endoscopy in international digestive endoscopy network 2012: how much has it advanced? Clin Endosc. 2012;45:297-8. Doi: https:// doi.org/10.5946/ce.2012.45.3.297

11. Shim ChS. How should biliary stones be managed? Gut and Liver. 2010;4(2):161-72. Doi: https://doi.org/10.5009/ gnl.2010.4.2.161

12. Ersoz G, Tekesin O, Ozutemiz A, et al. Biliary sphincterotomy plus dilation with a large balloon for bile duct stones that are difficult to extract. Gastrointest Endosc. 2003;57:156-9. Doi: https://doi.org/10.1067/mge.2003.52

13. Attam J, Freeman M. Endoscopic papillary large balloon dilation for large common bile duct stones. J Hepatobiliary Pancreat Surg. 2009;16:618-23. Doi: https://doi. org/10.1007/s00534-009-0134-2

14. Tae $\mathrm{H}$, Jin $\mathrm{K}$, Dong $\mathrm{S}$, et al. International consensus guidelines for endoscopic papillary large-balloon dilation. Gastrointest Endosc. 2016;83(1):37-47. Doi: https://doi. org/10.1016/j.gie.2015.06.016

15. Li G, Pang Q, Zhang X, et al. Dilation-assisted stone extraction: an alternative method for removal of common bile duct stones. Dig Dis Sci. 2014;59:857-64. Doi: https://doi.org/10.1007/s10620-013-2914-4

16. Jun Bo Q Li Hua X, Tian Min C, et al. Small endoscopic sphincterotomy plus large-balloon dilation for removal of large common bile duct stones during ERCP. PakJ Med Sci. 2013;29:90712. Doi: https://doi.org/10.12669/pjms.294.3662

17. Park SJ, Kim JH, Hwang JC, et al. Factors predictive of adverse events following endoscopic papillary large balloon dilation: results of a multicenter series. Dig Dis Sci. 2012;58:1100-9. Doi: https://doi.org/10.1007/s10620-012-2494-8

18. Kim JH, Yang MJ, Hwang JC, et al. Endoscopic papillary large balloon dilation for the removal of bile duct stones. World J Gastroenterol. 2013;19:8580-94. Doi: https://doi. org/10.3748/wjg.v19.i46.8580

19. Feng Y, Zhu H, Chen X, et al. Comparison of endoscopic papillary large balloon dilation and endoscopic sphincterotomy for retrieval of choledocholithiasis: a meta-analysis of randomized controlled trials. J Gastroenterol. 2012;47:65563. Doi: https://doi.org/10.1007/s00535-012-0644-6 / https://doi.org/10.1007/s00535-012-0528-9

20. Jin PP, Cheng JF, Liu D, et al. Endoscopic papillary large balloon dilation vs endoscopic sphincterotomy for retrieval of common bile duct stones: a meta-analysis. World J Gastroenterol. 2014;20:5548-56. Doi: https://doi. org/10.3748/wjg.v20.i18.5548

21. Madhoun MF, WaniS, Hong S, etal.Endoscopic papillarylarge balloon dilation reduces the need for mechanical lithotripsy in patients with large bile duct stones: a systematic review and meta-analysis. Diagn Ther Endosc. 2014;2014:309618. Doi: https://doi.org/10.1155/2014/309618

22. Yang XM, Hu B. Endoscopic sphincterotomy plus large-balloon dilation vs endoscopic sphincterotomy for choledocholithiasis: a meta-analysis. World J Gastroenterol. 2013;19:9453-60. Doi: https://doi.org/10.3748/wjg.v19.i48.9453

23. Liu Y, Su P, Lin Y, et al. Endoscopic sphincterotomy plus balloon dilation versus endoscopic sphincterotomy for choledocholithiasis: a meta-analysis. J Gastroenterol Hepatol. 2013;28:937-45. Doi: https://doi.org/10.1111/jgh.12192 